\title{
REVIEW \\ Molecular Identification and Evaluation of Orius species (Heteroptera: Anthocoridae) as Biological Control Agents
}

\author{
Norihide HINOMOTO ${ }^{1 *}$, Shigeyuki NAGAMORI ${ }^{2,4}$, Kazuki KAKIMOTO ${ }^{3,5}$, \\ Toru SHIMIZU ${ }^{1,6}$, Tomomi HIGAKI ${ }^{1}$, Masahiko MURAJI', Takashi NODA ${ }^{1}$ \\ and Kenjiro KAWASAKI ${ }^{\mathbf{1}}$
}

${ }^{1}$ Natural Enemies Laboratory, Insect Interaction Research Unit, National Institute of Agrobiological Sciences (Tsukuba, Ibaraki 305-8634, Japan)

${ }^{2}$ Agricultural Experiment Station, Okayama Prefectural General Agriculture Center

(Akaiwa, Okayama 709-0801, Japan)

${ }^{3}$ Kagoshima Prefectural Sericultural Experiment Station

(Higashi-ichiki, Kagoshima 899-2201, Japan)

\begin{abstract}
Orius strigicollis is a predatory bug that attacks small arthropods, such as thrips, and augmentative release of commercial strains has been widely conducted in greenhouses in Japan. The accurate evaluation of its effectiveness is essential for successful biological control programs. However, because Orius species occur naturally in the field, it is difficult to discriminate O. strigicollis from other Orius species. Furthermore, it is necessary to discriminate commercial strains of $O$. strigicollis from field populations. In this review, we introduce two types of molecular procedures: (1) a multiplex polymerase chain reaction technique to identify Japanese Orius species, and (2) microsatellite DNA markers that can be used to measure genetic diversity within populations of $O$. strigicollis and to discriminate field and commercial strains. The usefulness of molecular ecological approaches is also discussed.
\end{abstract}

Discipline: Insect pest / Plant protection

Additional key words: augmentative release, biopesticide, microsatellite DNA marker, natural enemy, polymerase chain reaction

\section{Introduction}

Chemical control of agricultural pests always poses a risk of them developing resistance to the pesticides. Therefore, integrated pest management (IPM) is essential for the control of pests, and augmentative release of natural enemies is one of the most important techniques for IPM ${ }^{29}$. In Japan, natural enemies must be registered as biopesticides when they are provided as commercial products, and 16 species were registered and sold as biopesticides in September, 2008. Although the registered species were exotic in the early stage of IPM, researchers have focused recently on the indigenous natural enemies to the pests, because such species are innately adapted to the environment in Japan and unexpected nontarget effects can be avoided by using indigenous species ${ }^{28}$.

The anthocorid bugs, particularly Orius spp. (Heteroptera: Anthocoridae), are the most promising biological control agents of various minute pests, such as thrips, aphids and mites ${ }^{15}$. Among seven species in the genus distributed in Japan, O. sauteri (L.), O. strigicollis (Poppius), O. minutus (L.), and O. nagaii Yasunaga are frequently found in the field ${ }^{30-32}$. Among these four species, $O$. sauteri has been investigated most often as a biological control agent of thrips, and its effectiveness in suppress-

\footnotetext{
Present address:

${ }^{4}$ Okayama Agricultural Improvement and Advisory Center (Okayama 700-8604, Japan)

${ }^{5}$ Soo Agricultural Improvement and Advisory Center (Soo, Kagoshima 899-8102, Japan)

${ }^{6}$ Ryukyu Sankei Co., Ltd. (Tomigusuku, Okinawa 901-0242, Japan)

*Corresponding author: e-mail hinomoto@affrc.go.jp

Received 1 October 2008; accepted 13 January 2009.
} 
ing thrips has been demonstrated both in the field and in greenhouses ${ }^{11,13,18,19}$. Because of the high incidence of reproductive diapause with short day length ${ }^{6,7,9,12,22}$ and low reproduction and predation rates at low temperatures ${ }^{8,18,19}$, however, $O$. sauteri is not suitable for use during the winter. In contrast, because of a lower incidence of reproductive diapause $\mathrm{e}^{9,22}$ and the ease of mass production, $O$. strigicollis was proposed as an effective biological control agent and was registered as a biopesticide in 2001 .

In biological control programs, it is essential to select appropriate species and evaluate released strains. These Orius species, however, are morphologically similar to each other and difficult to discriminate. Furthermore, released biopesticide strains cannot be distinguished easily from field populations. With the arrival of a vast array of molecular methods, however, it is now possible to develop a system to recognize different species and strains without morphological expertise ${ }^{24}$. In this article, we review molecular markers by which Orius species can be identified and evaluated, and describe our new understanding about the distribution of Orius species in Japan.

\section{Molecular identification of Orius species and its application}

\section{Molecular identification techniques}

Because several Orius species often occur sympatrically $^{21}$, proper identification of each species is necessary for the evaluation of their efficacy in biological control programs. Males are usually identified by their genitalia after dissection, but this process is time-consuming and identifies only adult males. Therefore, a polymerase chain reaction-restriction fragment length polymorphism (PCR-RFLP) method was developed to discriminate five Japanese Orius species ${ }^{4,17}$. This method is complicated, however, because restriction enzyme treatment is required after PCR amplification. Therefore, a multiplex PCR method, allowing simultaneous amplification of several fragments in a single PCR reaction, was developed based on the DNA sequences of nuclear ribosomal DNA by designing species-specific primers for the internal transcribed spacer regions ${ }^{3}$. Based on tests of 350 individuals of five Orius species (O. minutus, O. sauteri, O. strigicollis, $O$. nagaii, and $O$. tantillus), these species could be clearly distinguished by a single multiplex PCR reaction (Fig. 1). This technique is simple and requires no procedures other

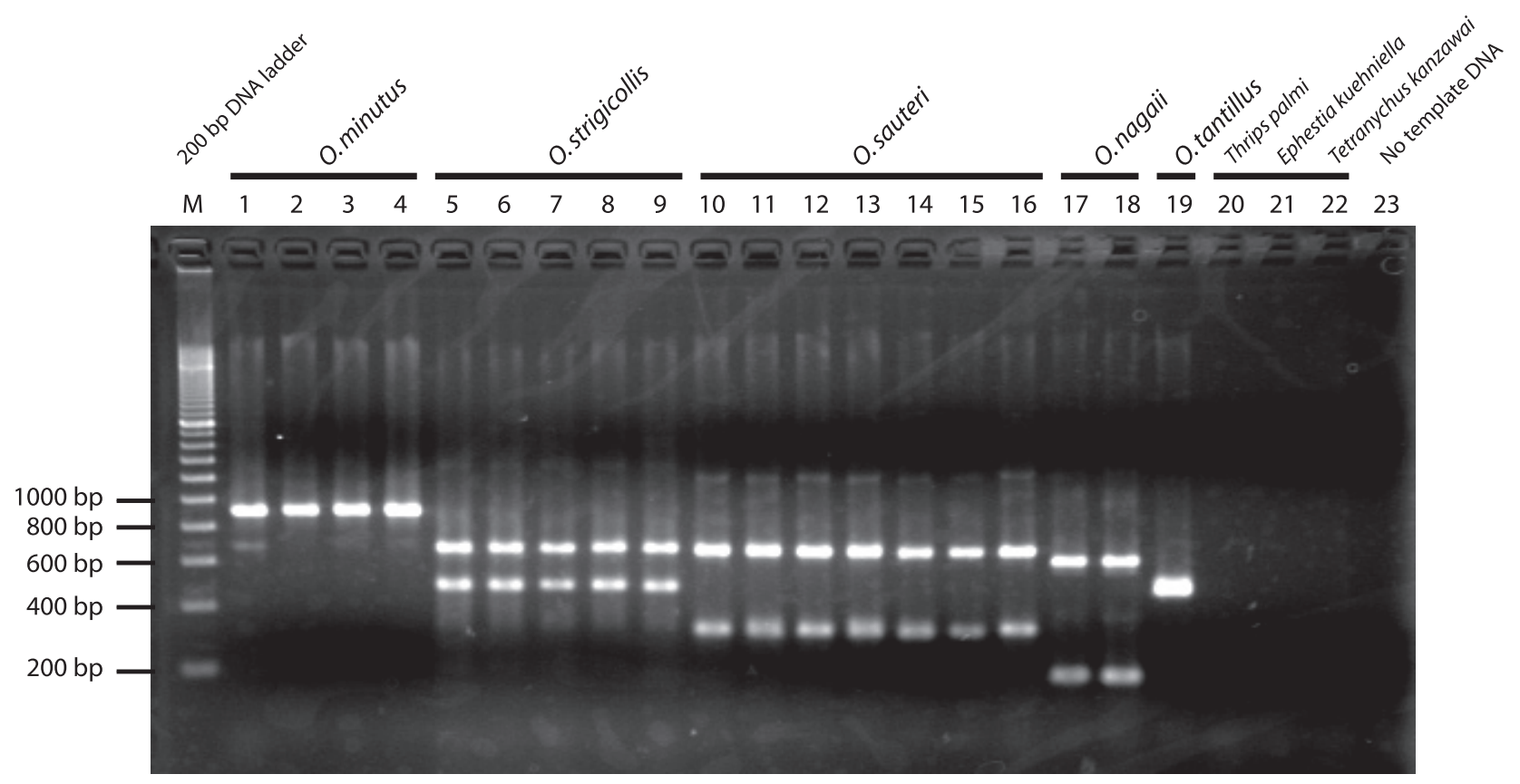

Fig. 1. Electrophoretogram of multiplex polymerase chain reaction for five Orius species and three prey species PCR products of a single insect were applied to each lane. Only O. minutus showed PCR products longer than $800 \mathrm{bp}$. Orius tantillus showed one band with a length of ca. $500 \mathrm{bp}$. Orius strigicollis, O. sauteri and O. nagaii showed two bands, one longer than $600 \mathrm{bp}$, and a shorter band that depended on the species: longer than $400 \mathrm{bp}$ (O. strigicollis), between 400 and $200 \mathrm{bp}$ (O. sauteri) and shorter than $200 \mathrm{bp}$ (O. nagaii). Prey species did not show any bands. M: molecular weight marker; 1-4: O. minutus; 5-9: O. strigicollis; 10-16: O. sauteri; 17-18: O. nagaii; 19: O. tantillus; 20: Thrips palmi; 21: Ephestia kuehniella; 22: Tetranychus kanzawai; 23: no template DNA3. 
than PCR and subsequent electrophoresis, thus reducing the cost of identifying species and increasing the number of specimens that can be identified.

\section{Geographic distribution of Orius strigicollis}

Among the four major Orius species, only the distribution of $O$. strigicollis is limited to southwestern Japan. However, their distribution areas and the factors limiting them have not been surveyed in detail. By using molecular discrimination techniques as well as morphological dissection, we identified Orius species collected on flowers of the tall goldenrod, Solidago altissima L., in Kanto district, east-central Japan, which is the northern limit of the distribution of $O$. strigicollis ${ }^{23}$. The factor determining this northern limit of $O$. strigicollis is the low temperature in winter ${ }^{23}$; the species is distributed in areas where the daily minimum temperature in January is higher than $-1^{\circ} \mathrm{C}$ on average and the number of days with a minimum temperature lower than $0^{\circ} \mathrm{C}$ was fewer than 50 days. Due to this limitation, O. strigicollis is distributed only in the coastal and urban areas in Kanto district.

In autumn from 2002 to 2007, we collected and identified Orius species from tall goldenrod flowers throughout southwestern Japan (Fig. 2). O. strigiciollis was found only in coastal and urban areas. Interestingly, O. strigiciollis is the dominant species in southern Kyushu but it is rarely distributed in northern Kyushu, where this species should be able to survive based on the temperature in winter ${ }^{23}$. Because O. strigicollis is distributed in the Okinawan islands, Taiwan and southern China as well ${ }^{31}$, the species may have originated in the south of Japan or more southern countries, with its distribution spreading northward with recent climate warming ${ }^{23}$

Conservation biological control, which manipulates the environments in agro-ecosystems to enhance natural enemies ${ }^{14}$, has recently attracted a great deal of attention in Japan. The detailed information on the distribution of Orius species described here will be necessary for the

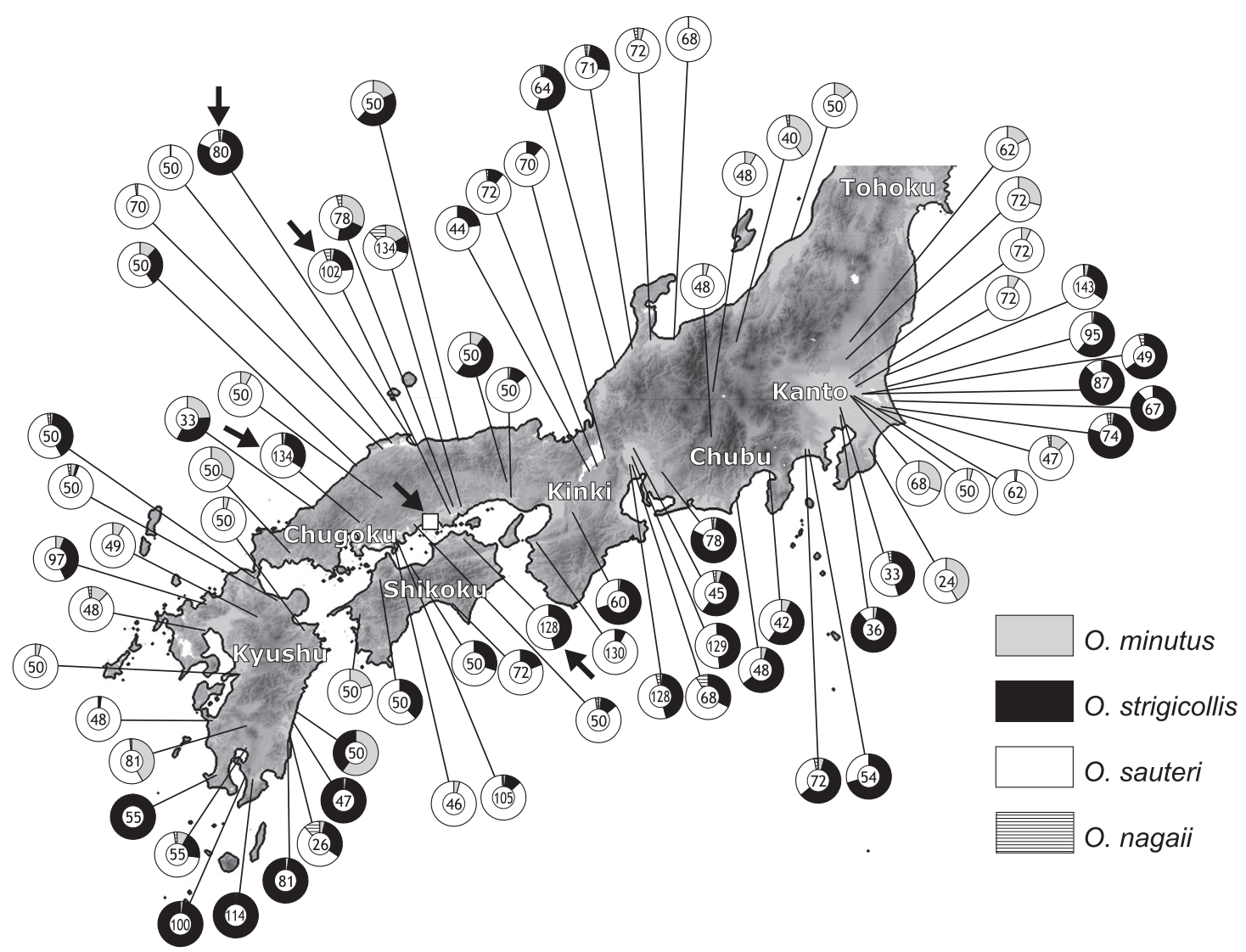

Fig. 2. Species composition of Orius collected from flowers of tall goldenrod, Solidago altissima, in autumn from 2002 to 2007

The numbers of Orius adults identified are shown in circles. Orius strigicollis was found only in coastal and urban areas in southwestern Japan. Arrows and the white square indicate field populations and the experimental sites (shown in Fig. 6). 
(a) O. strigicollis distribution area
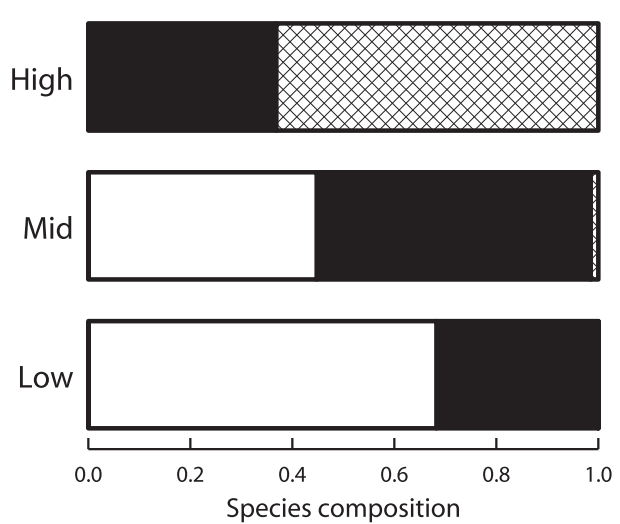

(b) O. strigicollis absent area

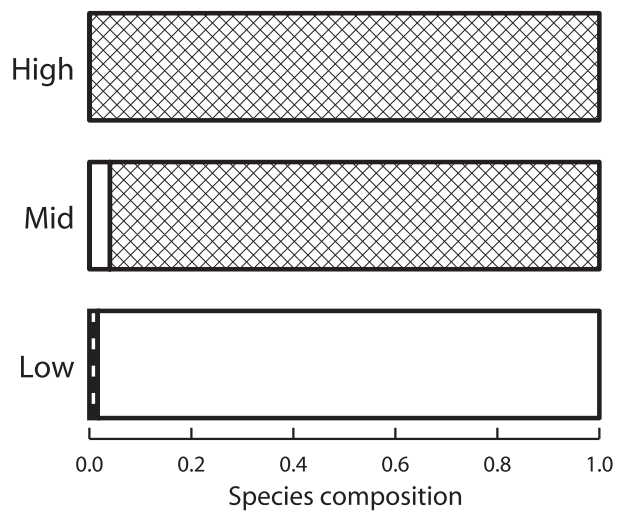

Fig. 3. Species composition of Orius collected from the various flowers

The composition was significantly different among the height of the flowers on which Orius species were collected (high: higher than $200 \mathrm{~cm}$ above the ground; mid: between 20 and $200 \mathrm{~cm}$; low: less than $20 \mathrm{~cm}$; Fisher's exact test; $p<0.0001$ ).

囚O.minutus, -O.strigicollis, $\square$ O.sauteri, $\equiv$ O.nagaii

proper conservation of these species and the management of their habitat.

\section{Vertical distribution of Orius species}

We collected Orius adults from flowers of a variety of plants and identified them by male genitalia or molecular techniques. Although O. sauteri, O. strigicollis, O. minutus, and $O$. nagaii can be found on a variety of plants, the microhabitats of each species seem to be different (Fig. 3). Orius sauteri adults were abundant on flowers at less than $20 \mathrm{~cm}$ above the ground, such as white clover (Trifolium repens L.). Orius minutus was mainly found on flowers higher than $200 \mathrm{~cm}$ above the ground, such as chestnut (Castanea crenata Sieb. et Zucc.) and apple (Malus pumila Mill.) trees, which was in accord with the previous observations $^{31}$. Orius strigicollis tended to inhabit flowers at intermediate heights above the ground between 20 and 200 $\mathrm{cm}$. The few $O$. nagaii individuals were found only on low flowers. The preference of these four species seems to have no relation to plants or prey species.

To clarify whether $O$. sauteri, O. strigicollis and $O$. minutus showed a differential distribution by height on the same plant species, we investigated the species composition at different heights on mulberry (Morus alba L.) trees. A mulberry field (ca. 4,000 $\mathrm{m}^{2}$ ) was divided into four sections; the heights of trees were adjusted to high (180-190 $\mathrm{cm})$ in two sections and low $(70-80 \mathrm{~cm})$ in the other sections by trimming. Orius individuals found within $15 \mathrm{~cm}$ of the top of newly growing shoots were collected and identified. The distribution of the three Orius species differed significantly among heights (Fig. 4).

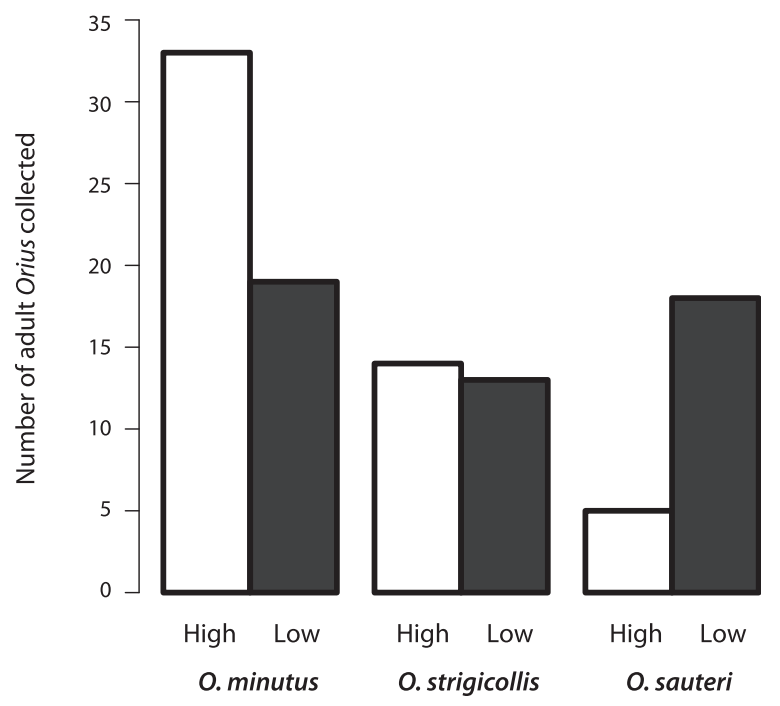

Fig. 4. Differential distribution of three Orius species at the different heights (high: $180-190 \mathrm{~cm}$; low: $70-80 \mathrm{~cm}$ ) of Morus alba

The distributions of the species were significantly different among heights ( $\chi^{2}$-test; $\left.p<0.01\right)$.

These findings allow the proposal of new usages of Orius as biological control agents. For example, O. sauteri has a good possibility for biological control on lowheight plants, and $O$. minutus would be a good candidate to control fruit tree pests. In fact, O. minutus was shown to control two-spotted spider mites, Tetranychus urticae Koch, in apple orchards ${ }^{25}$. These two species can play 
important roles in the situations where $O$. strigicollis cannot control pests effectively.

\section{Intraspecific DNA markers of Orius strigicollis}

\section{Microsatellite DNA markers}

Orius strigicollis is now widely used in Japan as a biological control agent in greenhouses ${ }^{29}$. Because O. strigicollis is common in the field of coastal and urban areas, it is difficult to judge the effectiveness of released strains in controlling pests. To discriminate among intraspecific strains of $O$. strigicollis, we developed six microsatellite DNA markers that distinguish field and commercial populations ${ }^{2}$

When genetic diversity within each field population collected on the tall goldenrod were tested, the inbreeding coefficient $^{27}\left(F_{\text {IS }}\right)$ was significantly different from zero, whereas biopesticide populations did not show such deviation. This indicated that the field populations of $O$. strigicollis would generally show heterozygote deficits. Orius adults appear to immigrate into tall goldenrod habitats in late autumn, attracted by herbivorous insects crowding on the flowers ${ }^{23}$. Thus, the heterozygote deficits might result from population genetic structures, such as the Wahlund effect.

Genetic differentiation among populations was estimated by the fixation index ${ }^{27}\left(F_{\mathrm{ST}}\right)$ values. Field populations collected in geographically close areas showed no genetic differentiation. In contrast, two biopesticide strains were markedly differentiated genetically from any field populations, revealing the high genetic specificity of these strains. Specific alleles for biopesticide populations would be very useful for distinguishing released biopesticide from field populations. No such specific alleles were found, however, probably because the commercial O. strigicollis populations were derived from somewhere in Japan.

Biopesticide populations were also characterized by significantly less allelic richness than that of field populations (Fig. 5). The biopesticide populations might have lost genetic diversity through bottleneck effects and genet- ic drift during laboratory rearing, as well as through possible selection during mass production. The importance of quality control in mass-production procedures of biological control agents has been recognized, and the genetic structure of laboratory populations is one of the most important factors ${ }^{1}$. When several strains are reared, contamination of different strains should be avoided. Some genetic effects such as bottleneck and genetic drift often reduce or alter the attributes of reared populations. By quantifying genetic composition within populations, such deterioration can be easily monitored.

\section{Post-release evaluation}

The effectiveness of $O$. strigicollis as a biological control agent released in a greenhouse was evaluated by microsatellite markers ${ }^{20}$. Eggplants were planted in October 2002, and the population dynamics of Orius and thrips were monitored until June 2003. A commercial strain of O. strigicollis was released three times in the greenhouse where an IPM program was conducted. In contrast to the insecticide-sprayed greenhouse, in which thrips could not be controlled after March, the density of thrips was at a low level in the IPM greenhouse from October to June. After the harvest, Orius collected in the greenhouses and on weeds outside were identified by the multiplex PCR method (Table 1). In the IPM greenhouse, almost all the Orius individuals were $O$. strigicollis, and in the insecticide-sprayed greenhouse the dominant species was also O. strigicollis. Outside of the greenhouses, however, the most dominant species was $O$. nagaii, followed by $O$. sauteri. In this case, the O. strigicollis collected in the IPM greenhouse should be descendants of the released strain; however, it is also possible that wild $O$. strigicollis intruded into the greenhouses. We compared the microsatellite allele frequencies of $O$. strigicollis collected from three experimental sites (the IPM greenhouse, insecticide-sprayed greenhouse and weedy area), four field populations in this district (indicated by arrows in Fig. 2), and a released biopesticide population. The three experimental populations were genetically very close to the biopesticide population, whereas the field populations were significantly different

Table 1. Species composition of Orius collected at three experimental sites, where a biopesticide strain was released in a greenhouse to control thrips on eggplants ${ }^{20}$

\begin{tabular}{lcccc}
\hline \hline Site & O. strigicollis & O. sauteri & O. nagaii & $\mathrm{n}^{*}$ \\
\hline IPM greenhouse & $91.7 \%$ & $8.3 \%$ & $0.0 \%$ & 24 \\
Insecticide-sprayed greenhouse & $79.4 \%$ & $17.6 \%$ & $2.9 \%$ & 34 \\
Weedy area & $29.0 \%$ & $32.3 \%$ & $38.7 \%$ & 31 \\
\hline
\end{tabular}

$*_{n}$ : Total number of Orius adults collected. 

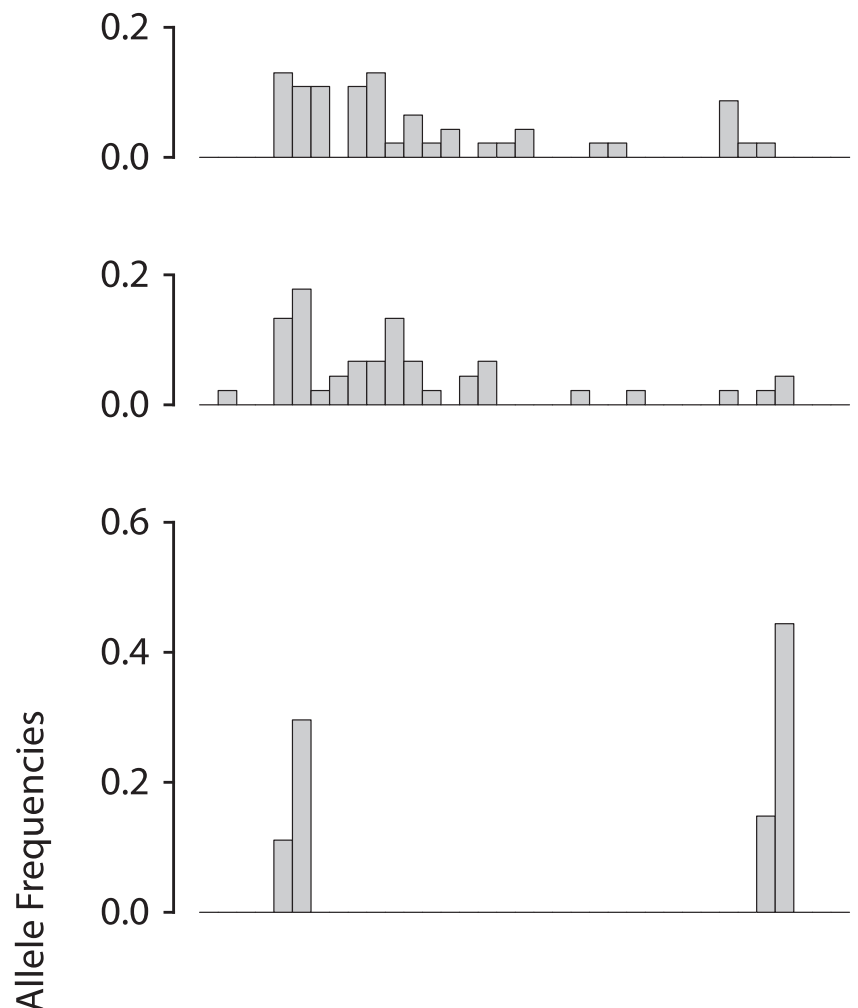

Miyazaki Field population

Kanoya1

Field population

Tairiku ${ }^{\circledast}$

Biopesticide

Oristar-A ${ }^{\oplus}$

Biopesticide

\section{Allele Code}

Fig. 5. Example of allele frequencies of a microsatellite marker in Orius strigicollis

Allele frequencies of OstMSO09 in two fields and two commercial populations are shown. The number of alleles detected was greater in the field populations than in the biopesticide populations. In a biopesticide Oristar- $\mathrm{A}^{\circledR}$ (Sumitomo Chemical, Co. Ltd.), no polymorphisms were detected in this locus ${ }^{2}$. 


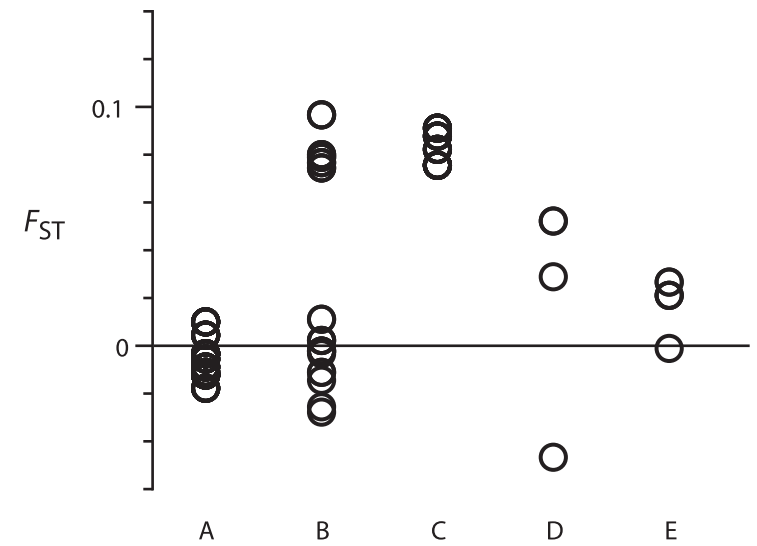

Fig. 6. Pairwise fixation index $\left(F_{\mathrm{ST}}\right)$ among Orius strigicollis populations

A: among field populations (collection sites are shown in Fig. 2); B: between field and experimental sites; $\mathrm{C}$ : between field populations and a biopesticide population; D: among experimental sites; E: between experimental sites and a biopesticide population. When $F_{\mathrm{ST}}$ is zero, the populations compared are considered to be genetically identical ${ }^{20}$.

(Fig. 6). Therefore, the Orius strain collected in the IPM greenhouse, which successfully controlled thrips, was not a field strain but the released biopesticide strain. These results indicated that the preservation of released Orius by reducing insecticide spray was important in controlling thrips in the IPM program.

\section{Conclusion and future remarks}

Biological control programs often fail because of the lack of understanding about the fundamental biology of the biological control agents or its target pests, including the population genetic structure, dynamics, overwintering, reproduction, and host preference ${ }^{16}$. Identification of species is the first step in understanding such factors, but this is sometimes difficult due to the agents' small size. The advent of PCR, however, has allowed amplification of molecular markers from small amounts of DNA, even from a single insect egg.

Molecular markers can also help us to evaluate the effect of biological control agents at the intraspecific level. For example, although small arthropods cannot be observed directly during a long-term cultivation period, inherited genetic markers can enable us to monitor them over generations. Microsatellite DNA markers have been widely employed in molecular ecological studies because their high polymorphism makes the markers suitable for studying intraspecific variation. However, microsatellite markers are difficult to isolate from the arthropod genome, so that the molecular ecological approach has not been established in biological control. Nevertheless, through the recent development of isolation techniques ${ }^{33}$, microsatellite markers have been developed for biological control agents, especially in parasitoid wasps ${ }^{5,10,26}$

By using molecular markers, the uncertain factors that previously caused difficulties in biological control programs can be measured quantitatively. We hope that the molecular ecological approach reviewed here will be developed and used more widely for the further development of IPM programs.

\section{Acknowledgments}

Our studies reviewed in this article were supported by the Experimental Research Budget on Pollution Prevention and Natural Environmental Conservation from the Ministry of the Environment, Japan; Grant-in-Aid for Young Scientists (B) (no. 18780040) from the Ministry of Education, Culture, Sports, Science and Technology, Japan; and the Research Project (Selection of Functional Diversity Indicators and Development of Assessment Methods) from the Ministry of Agriculture, Forestry and Fisheries, Japan.

\section{References}

1. Bigler, F. (1989) Quality assessment and control in entomophagous insects used for biological control. J. Appl. Entomol., 108, 390-400.

2. Hinomoto, N., Higaki, T. \& Noda, T. (2006) Genetic diversity in field and commercial populations of Orius strigicollis (Poppius) (Heteroptera: Anthocoridae) measured by microsatellite markers. Appl. Entomol. Zool., 41, 499-506.

3. Hinomoto, N. et al. (2004) Identification of five Orius species in Japan by multiplex polymerase chain reaction. Biol. Control, 31, 276-279.

4. Honda, J. Y. et al. (1999) Isoelectric focusing electrophoresis and RFLP analysis: two methods for immature Orius spp. identification. Appl. Entomol. Zool. 34, 69-74.

5. Hufbauer, R. A. et al. (2001) Isolation and characterization of microsatellites in Aphidius ervi (Hymenoptera: Braconidae) and their applicability to related species. Mol. Ecol. Notes, 1, 197-199.

6. Ito, K. \& Nakata, T. (1998) Effect of photoperiod on reproductive diapause in the predatory bugs, Orius sauteri (Poppius) and O. minutus (Linnaeus) (Heteroptera: Anthocoridae). Appl. Entomol. Zool., 33, 115-120.

7. Ito, K. \& Nakata, T. (2000) Geographical variation of photoperiodic response in the females of a predatory bug, Orius sauteri (Poppius) (Heteroptera: Anthocoridae) from northern Japan. Appl. Entomol. Zool., 35, 101-105.

8. Kakimoto, K. et al. (2005) Comparison of the reproductive potential of three Orius species, O. strigicollis, O. sauteri, and $O$. minutus (Heteroptera: Anthocoridae), using eggs of the Mediterranean flour moth as a food source. Appl. 
Entomol. Zool., 40, 247-255.

9. Kakimoto, K., Hinomoto, N. \& Noda, T. (2003) Responses of three Orius species collected in Kagoshima to different rearing temperatures and photoperiods. Jpn. J. Appl. Entomol. Zool., 47, 19-28 [In Japanese with English summary].

10. Kankare, M. \& Shaw, M. R. (2004) Molecular phylogeny of Cotesia Cameron, 1891 (Insecta: Hymenoptera: Braconidae: Microgastrinae) parasitoids associated with Melitaeini butterflies (Insecta: Lepidoptera: Nymphalidae: Melitaeini). Mol. Phylogenet. Evol., 32, 207-220.

11. Kawai, A. (1995) Control of Thrips palmi Karny (Thysanoptera: Thripidae) by Orius spp. (Heteroptera: Anthocoridae) on greenhouse eggplant. Appl. Entomol. Zool., 30, 1-7.

12. Kohno, K. (1998) Thermal effects on reproductive diapause induction in Orius sauteri (Heteroptera: Anthocoridae). Appl. Entomol. Zool., 33, 487-490.

13. Kohno, K. \& Kashio, T. (1998) Development and prey consumption of Orius sauteri (Poppius) and O. minutus (L.) (Heteroptera: Anthocoridae) fed on Frankliniella occidentalis (Pergande) (Thysanoptera: Thripidae). Appl. Entomol. Zool., 33, 227-230.

14. Landis, D. A., Wratten, S. D. \& Gurr, G. M. (2000) Habitat management to conserve natural enemies of arthropod pests in agriculture. Annu. Rev. Entomol., 45, 175-201.

15. Lattin, J. D. (1999) Bionomics of the anthocoridae. Annu. Rev. Entomol., 44, 207-231.

16. MacDonald, C. \& Loxdale, H. D. (2004) Molecular markers to study population structure and dynamics in beneficial insects (predators and parasitoids). Int. J. Pest Manag., 50, 215-224.

17. Muraji, M. et al. (2004) Discrimination among Japanese species of the Orius flower bugs (Heteroptera: Anthocoridae) based on PCR-RFLP of the nuclear and mitochondrial DNAs. JARQ, 38, 91-95.

18. Nagai, K. \& Yano, E. (1999) Effects of temperature on the development and reproduction of Orius sauteri (Poppius) (Heteroptera: Anthocoridae), a predator of Thrips palmi Karny (Thysanoptera: Thripidae). Appl. Entomol. Zool., 34, 223-229.

19. Nagai, K. \& Yano, E. (2000) Predation by Orius sauteri (Poppius) (Heteroptera: Anthocoridae) on Thrips palmi Karny (Thysanoptera: Thripidae): Functional response and selective predation. Appl. Entomol. Zool., 35, 565-574.

20. Nagamori, S. \& Hinomoto, N. (2004) Effectiveness of Orius strigicollis (Poppius) (Hemiptera: Anthocoridae) released in autumn against Thrips palmi Karney (Thysanoptera: Thripidae) in the forcing culture of eggplant. Bull. Agr. Exp. Station, Okayama Prefectural General Agriculture Center, 22, 59-63 [In Japanese with English summary].
21. Ohno, K. \& Takemoto, H. (1997) Species composition and seasonal occurrence of Orius spp. (Heteroptera: Anthocoridae), predacious natural enemies of Thrips palmi (Thysanoptera: Thripidae) in eggplant fields and surrounding habitats. Appl. Entomol. Zool., 32, 27-35.

22. Shimizu, T. \& Kawasaki, K. (2001) Geographic variability in diapause response of Japanese Orius species. Entomol. Exp. Appl., 98, 303-316.

23. Shimizu, T., Kawasaki, K. \& Hinomoto, N. (2001) Factors limiting the northern distribution in Orius strigicollis (Poppius) (Heteroptera: Anthocoridae). Jpn. J. Entomol. (n.s.), 4, 129-141 [In Japanese with English summary].

24. Stouthamer, R. (2006) Molecular methods for the identification of biological control agents at the species and strain level. In Environmental impact of invertebrates for biological control of arthropods: Methods and risk assessment, eds. Bigler, F., Babendreier, D. \& Kuhlmann, U., CABI Publishing, Wallingford, UK, 187-201.

25. Toyoshima, S. \& Osakabe, M. (2005) Effects of artificially released Neoseiulus californicus (Acari: Phytoseiidae) and naturally occurring Orius minutus (Hemiptera: Anthocoridae) on Tetranychus urticae (Acari: Tetranychidae) population in apple orchard without insecticides. Annu. Rep. Soc. Plant Prot. N. Jpn., 56, 188-190 [In Japanese with English summary].

26. Vanlerberghe-Masutti, F. \& Chavigny, P. (1997) Characterization of a microsatellite locus in the parasitoid wasp Aphelinus abdominalis (Hymenoptera: Aphelinidae). Bull. Entomol. Res., 87, 313-318.

27. Weir, B. S. \& Cockerham, C. C. (1984) Estimating $F$ statistics for the analysis of population structure. Evolution, 38, 1358-1370.

28. Yano, E. (1999) Recent advances in the study of biocontrol with indigenous natural enemies in Japan. IOBC Bull., 22, 291-294.

29. Yano, E. (2004) Recent development of biological control and IPM in greenhouses in Japan. J. Asia-Pacific Entomol., 7, 5-11.

30. Yasunaga, T. (1997a) The flower bug genus Orius Wolff (Heteroptera: Anthocoridae) from Japan and Taiwan, Part I. Appl. Entomol. Zool., 32, 355-364.

31. Yasunaga, T. (1997b) The flower bug genus Orius Wolff (Heteroptera: Anthocoridae) from Japan and Taiwan, Part II. Appl. Entomol. Zool., 32, 379-386.

32. Yasunaga, T. (1997c) The flower bug genus Orius Wolff (Heteroptera: Anthocoridae) from Japan and Taiwan, Part III. Appl. Entomol. Zool., 32, 387-394.

33. Zane, L., Bargelloni, L. \& Patarnello, T. (2002) Strategies for microsatellite isolation: a review. Mol. Ecol., 11, $1-16$. 